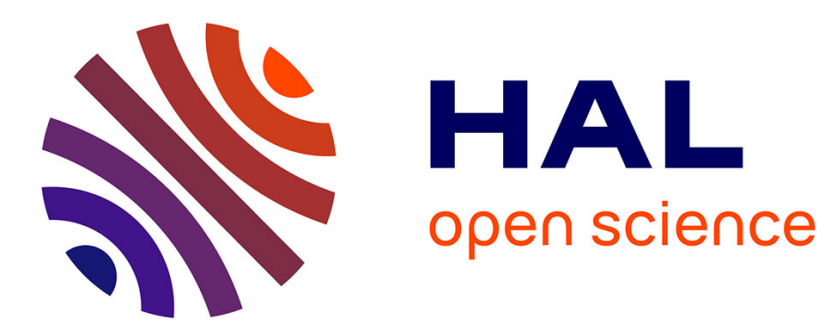

\title{
Investigation of the interaction between hydrogen and screw dislocation in silicon by first principles calculations
}

Laurent Pizzagalli

\section{To cite this version:}

Laurent Pizzagalli. Investigation of the interaction between hydrogen and screw dislocation in silicon by first principles calculations. Journal of Physics: Condensed Matter, 2009, 22, pp.035803. 10.1088/0953-8984/22/3/035803 . hal-00728916

\section{HAL Id: hal-00728916 https://hal.science/hal-00728916}

Submitted on 7 Sep 2012

HAL is a multi-disciplinary open access archive for the deposit and dissemination of scientific research documents, whether they are published or not. The documents may come from teaching and research institutions in France or abroad, or from public or private research centers.
L'archive ouverte pluridisciplinaire HAL, est destinée au dépôt et à la diffusion de documents scientifiques de niveau recherche, publiés ou non, émanant des établissements d'enseignement et de recherche français ou étrangers, des laboratoires publics ou privés. 


\title{
Investigation of the interaction between hydrogen and screw dislocation in silicon by first principles calculations
}

\author{
M Matsubara, J Godet and L Pizzagalli \\ Laboratoire PHYMAT, CNRS UMR 6630, Université de Poitiers, B.P. 30179, 86962 \\ Futuroscope Chasseneuil Cedex, France \\ E-mail: Laurent.Pizzagalli@univ-poitiers.fr
}

\begin{abstract}
.
The stability of atomic and molecular hydrogen in the vicinity of a screw dislocation in silicon has been investigated using first-principles calculations. Lowest energy configurations are obtained for $\mathrm{H}$ atoms located into the dislocation core, suggesting that the segregation of hydrogen is favoured into the dislocation core. It is found that a spontaneous dissociation of $\mathrm{H}_{2}$ could occur in the dislocation core. Finally, the variation of the interaction energy between hydrogen and the dislocation core as a function of the separating distance has been calculated. There is no sizeable interaction variation for $\mathrm{H}_{2}$. However, in the case of a single $\mathrm{H}$, an inverse law has been obtained which can be explained by the anisotropic stress field generated by the insertion of $\mathrm{H}$ in the silicon lattice.
\end{abstract}

PACS numbers: 61.72.Lk, 31.15.E-, 67.63.-r, 61.72.uf

Submitted to: J. Phys.: Condens. Matter 


\section{Introduction}

There have been a large number of investigations concerned with the behaviour of hydrogen in silicon (for a review see for instance [1]). This interest was largely driven by the potential technological applications, since hydrogen has been shown to interact with a wide range of defects and impurities, in particular dopants. In fact, hydrogen has the ability to passivate some electrically or optically active impurities, while activating normally inert defects. Structural defects, from the most simple one, the vacancy, to the surface, are typically characterized by the presence of dangling bonds, which strongly interact with hydrogen. In addition to modifying defect properties in silicon, it has also been demonstrated that hydrogen could be used to generate new defects. In fact, $\mathrm{H}$ implantation could lead to the formation of finite planar defects, the so-called hydrogen induced platelets $[2,3]$, used in the 'smart-cut' process for wafer bonding, as well as of bubbles filled with molecular hydrogen [4].

Because of the formidable ability of hydrogen to interact with defects, it is extremely difficult to determine fundamental properties associated with a single $\mathrm{H}$ atom in silicon, such as solubility and diffusion for instance. One of the earliest diffusion measurement has been performed by Van Wieringen and Warmoltz [5], yielding an activation energy for the diffusion of atomic hydrogen equal to $0.48 \mathrm{eV}$ at room temperature. There have been many other experimental [1] and theoretical [6, 7, 8, 9, 10] determinations afterwards, which all pointed that atomic hydrogen is highly mobile in silicon. Also, there is a general agreement that the lowest energy configuration is obtained for $\mathrm{H}$ located in a bond-centered (BC) position. However, an accurate determination of $\mathrm{H}$ diffusivity remains difficult, since it depends on many factors such as the charge state of $\mathrm{H}$, the doping of silicon, or the temperature. The formation of molecular hydrogen from single $\mathrm{H}$ atoms is energetically favoured [11, 12], with the most stable configuration corresponding to $\mathrm{H}_{2}$ located in a tetrahedral ( $\mathrm{T}$ ) site $[12,13]$. Much less information is available for $\mathrm{H}_{2}$ migration energy, although values of $1.74 \mathrm{eV}$ [13] and $1.1 \mathrm{eV}[14]$ have been proposed from first-principles calculations. $\mathrm{H}$ or $\mathrm{H}_{2}$ are expected to strongly interact with many possible structural defects in silicon, such as point defects, or surfaces $[11,15]$. For instance, it has been shown that both vacancies and interstitials could dissociate $\mathrm{H}_{2}$ with a substantial gain in energy [16].

Point defects, either intrinsic (vacancies, interstitials) or extrinsic (impurities such as dopants), are expected to interact with linear defects like dislocations. In the case of silicon, most of the studies were concerned with the effect of dopants on structural and electronic properties of dislocation cores [17, 18, 19, 20]. The interplay between vacancy cluster and undissociated $60^{\circ}$ dislocation has also been recently studied [21, 22]. However, to our knowledge, there have been relatively few investigations of the interaction between hydrogen and dislocations in silicon so far. Experimentally, it has been shown that the exposure to $\mathrm{H}$ plasma leads to a large reduction of the activation energy for dislocation mobility [23]. Ewels and co-workers have proposed an explanation for this so-called hydrogen enhanced dislocation glide effect in the framework 
of the soliton model $[24,25]$. In particular, they have shown that the formation of a kink pair on a $90^{\circ}$ partial dislocation releases energy if it occured at a $\mathrm{H}$-saturated soliton. Also, the energy barrier for soliton migration increases in presence of hydrogen.

Despite the latter works, the lack of available data does not allow for determining the interaction between hydrogen and dislocations in silicon in a more general perspective, and several questions remain unanswered. One may wonder whether hydrogen is effectively attracted to the dislocation core, and if yes to what extent? Is hydrogen segregation in the dislocation core likely to occur, and if yes, in the form of atomic $\mathrm{H}$ or molecular $\mathrm{H}_{2}$ ? Finally, it would be interesting to check whether the mobility of hydrogen along the dislocation line is modified compared to diffusion in bulk. Our aim in this work was to answer several of these questions, by computing the interaction between $\mathrm{H}$ or $\mathrm{H}_{2}$, and a screw dislocation in silicon. In the following, after describing the models and the computational methods, we show the most stable configurations for $\mathrm{H}$ and $\mathrm{H}_{2}$ in the dislocation core. Then, the variation of the interaction between hydrogen and the dislocation as a function of the separation is reported and discussed.

\section{Models and methods}

Our model for describing the interaction between screw dislocation and hydrogen is built according to the following procedure. First, a screw dislocation is introduced in bulk silicon, using displacements given by anisotropic elasticity theory and the experimental lattice constants. From these, systems with cylindrical shape and centered on the dislocation core are cut. The cylinder axis is orientated along the [101] direction, which is the screw dislocation line, along which a periodic boundary condition is applied for modeling an infinite straight dislocation. Conversely, along the two other directions, $[121]$ and $[\overline{1} 1 \overline{1}]$, i.e. perpendicular to the dislocation line, the system is finite (cluster-like conditions). Silicon atoms at the cylinder surface, defined as undercoordinated atoms, are passivated with hydrogen atoms, and are not allowed to relax during calculations, in order to retain the anisotropic elastic displacements field. Bulk-like atomistic models, i.e. without dislocations, have also been built for reference energy calculations.

Several possible core structures have been proposed for the non-dissociated screw dislocation. Among these, the lowest energy configuration is obtained when the center of the dislocation is located at the intersection of two (111) planes of the 'glide' set, with a double period reconstruction along the dislocation line [26, 27]. Another possible core configuration is obtained when the center of the dislocation is at the intersection of two (111) planes of the 'shuffle' set [28, 29]. This 'shuffle' core is stable, albeit with a larger energy than the 'glide' core. It is still not clear which one of these configurations are predominant during low temperature plasticity of silicon [30, 31, 32]. In this work, we used the shuffle core for the screw dislocation configuration, because its structure is more simple than the glide core (figure 1). Finally, atomic $\mathrm{H}$ or molecular $\mathrm{H}_{2}$ hydrogen is introduced at various locations in the system, corresponding to different separations $d$ with the screw dislocation center. The effect of the charge of hydrogen has not been 


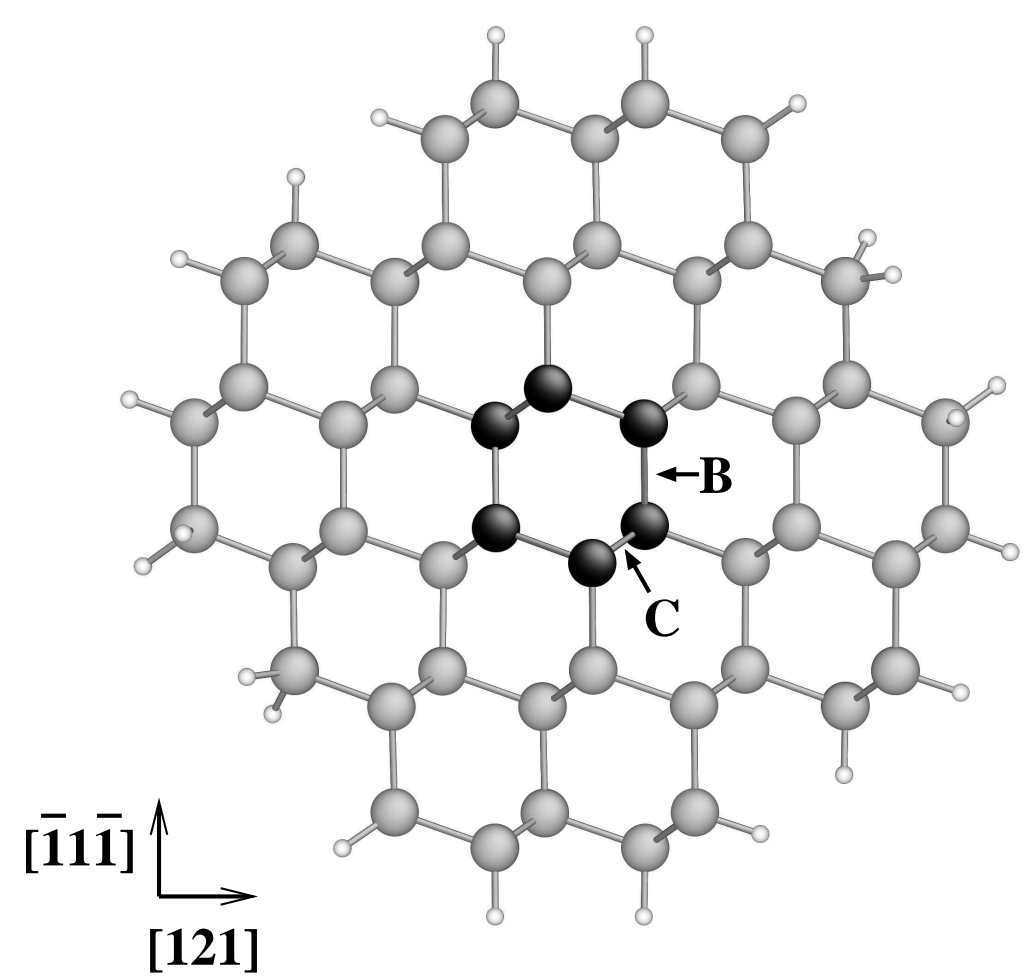

Figure 1. Ball-stick representation (projection along [101], the dislocation line) of the system used in the calculations. The screw dislocation line is located in the central hexagon, marked by black atoms. Small white spheres represent hydrogen atoms.

considered.

The calculations are based on density functional theory with generalized gradient approximations, which is implemented in the SIESTA code $[33,34,35]$. We have used the exchange and correlation functional after Perdew, Burke and Ernzerhof [36], and norm conserving pseudopotentials for ionic interactions. Wavefunctions are described with a generalized version of linear combinations of atomic orbitals, which include multiplezeta orbitals and polarization states. We have used single-zeta basis sets for $\mathrm{H}$ atoms passivating dangling bonds of surface Si atoms and double-zeta plus polarized basis sets for silicon and hydrogen atoms interacting with the dislocation. Finally, the charge density is projected onto a real-space grid with an equivalent cutoff of $40 \mathrm{Ry}$ [35]. Within these conditions, an optimized lattice constant $a_{0}$ of $5.484 \AA$, in good agreement with experimental data [37], is obtained. Note that calculations for a single $\mathrm{H}$ atom interacting with the screw have been performed with and without spin polarization, without any noticeable differences.

Our simulations have been performed in a supercell with a computational system encompassing at least 296 atoms, $200 \mathrm{Si}$ and $96 \mathrm{H}$ surface atoms (Figure 1). The dimension along the dislocation line is $4|\boldsymbol{b}|, \boldsymbol{b}=a_{0} / 2[\overline{101}]$ being the Burgers vector 
Table 1. Formation energy differences in eV per $\mathrm{H}$ atom for different $\mathrm{H}$ configurations in bulk silicon, relative to $\mathrm{H}_{2}$ in a $\mathrm{T}$ site, calculated with the equation 3 according to the formalism described in [12].

\begin{tabular}{lll}
\hline & $\delta$ E per $\mathrm{H}(\mathrm{eV})$ & {$[12]$} \\
\hline $\mathrm{H}_{2}$ in $\mathrm{T}$ & 0 & 0 \\
$\mathrm{H}_{2}^{*}$ & 0.09 & 0.10 \\
$\mathrm{H}$ in $\mathrm{BC}$ & 1.07 & 1.04 \\
\hline
\end{tabular}

of the dislocation. Along the other directions, perpendicular to the dislocation line, dimensions of $5 a_{0}$ have been used, yielding a vacuum of about $8 \AA$ between periodic images. Due to the large size of the system, the Brillouin zone is sampled at the $\Gamma$ point only. The cluster is relaxed by minimizing the forces on all atoms using conjugate gradient method, except for $\mathrm{Si}$ atoms at the surface and $\mathrm{H}$ atoms for dangling bonds saturation. The optimization of the ionic positions is allowed to proceed until the maximum atomic force is less than $0.005 \mathrm{eV} / \AA$.

First, it is interesting to check the accuracy of our model and computational procedure for an isolated screw dislocation, and for $\mathrm{H}$ and $\mathrm{H}_{2}$ interstitial in a bulklike system, i.e. without dislocation. In the first case, we found that the shuffle screw dislocation core is stable. Along [101], at location C (figure 1), there are short and long bonds, with lengths of $2.30 \AA$ and $2.54 \AA$ respectively, to be compared with the bulk bond length of $2.38 \AA$. At location $\mathrm{B}$, the bonds have lengths equal to $2.45 \AA$, with a tilting angle of $21^{\circ}$. These structural characteristics have been compared to a similar configuration relaxed in fully periodic systems with plane waves first principles calculations [28]. We found bond length differences lower than $1 \%$, indicating that our model allows a very good modelling of the screw dislocation. In the second case, we computed the formation energy differences per $\mathrm{H}$ atom, for $\mathrm{H}_{2}$ in the $\mathrm{T}$ site or in the $\mathrm{H}_{2}^{*}$ configuration [8], and for $\mathrm{H}$ in $\mathrm{BC}$ site. The results are reported in the table 1 , and compared with the calculations from Morris et al. [12]. The excellent agreement between values confirms that our computational method is well suited for the calculations of hydrogen defects in silicon.

\section{Stable configurations for $\mathbf{H}$ and $\mathbf{H}_{2}$ in the dislocation core}

It is useful to decompose dislocations into two defined regions. The first one, the dislocation core, is spatially confined and usually characterized by large atomic displacements that can not be described by elasticity theory. The second region surrounds the core, and features small displacements relatively to the perfect lattice, which are well described by elasticity theory. The boundary between these two domains is not clearly defined, and depends on the dislocation characteristics, as well as the material considered. It should not be confused with the so-called dislocation radius as defined by Hirth and Lothe, which is obtained by matching the excess energy due to 


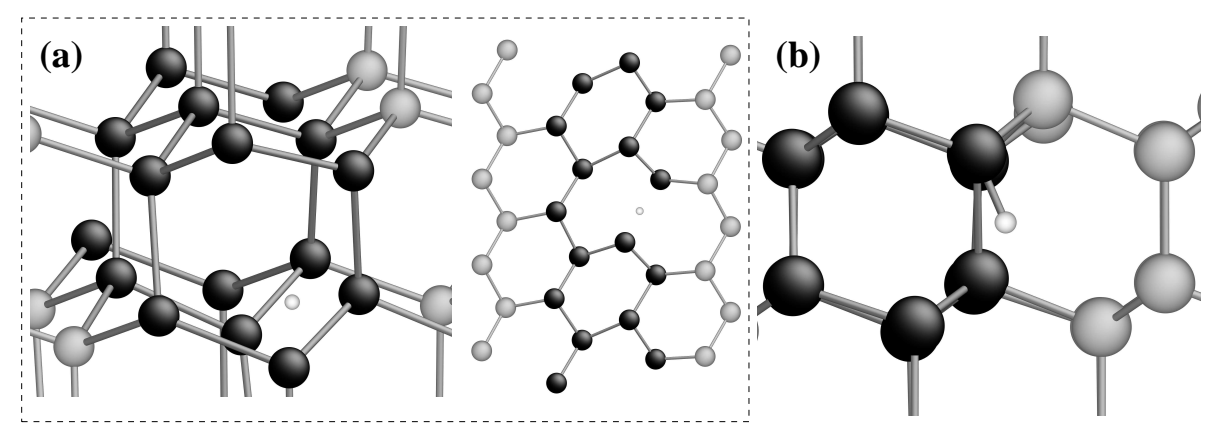

(c)

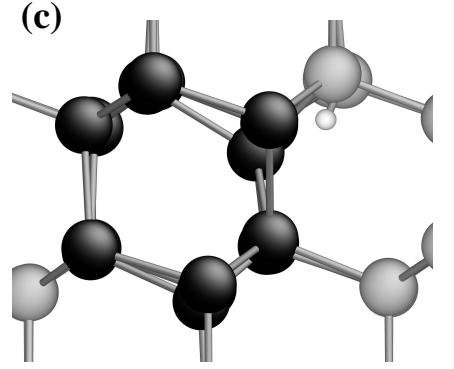

(d)

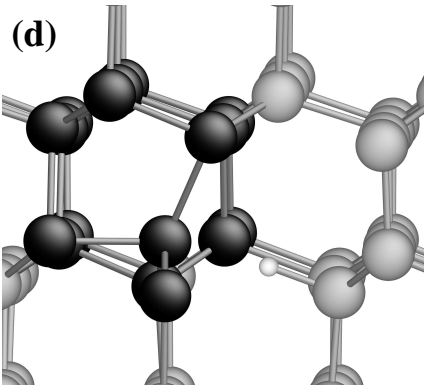

Figure 2. (a-d) Four possible stable configurations for a single $\mathrm{H}$ atom located in a screw dislocation core. The dislocation core is marked by black atoms. In the case of

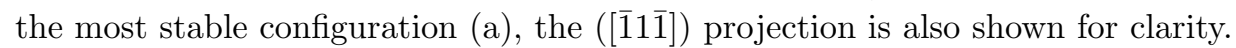

the dislocation with the elastic energy [38].

In the elastic region, i.e. far from the dislocation core, one can expect that stable hydrogen positions will be very close to bulk configurations, such as the BC site. Conversely, in the core, since the geometry can be very different from the ideal lattice, a systematic search for all stable configurations of hydrogen is required. We have first performed such an investigation for a single $\mathrm{H}$ starting from high-symmetry initial configurations, or for hydrogen positions close to bulk-like $\mathrm{BC}$ and $\mathrm{T}$ sites, or even for random configurations. For each relaxed configuration, we computed the defect formation energy for $\mathrm{H}$ or $\mathrm{H}_{2}$, defined as

$$
\begin{aligned}
& E_{F}^{\mathrm{H} / \perp}=E_{\circ}^{\mathrm{H} / \perp}-E_{\circ}^{\perp}-E_{\mathrm{H}}, \\
& E_{F}^{\mathrm{H}_{2} / \perp}=E_{\circ}^{\mathrm{H}_{2} / \perp}-E_{\circ}^{\perp}-2 E_{\mathrm{H}} .
\end{aligned}
$$

$E_{\circ}^{\mathrm{H} / \perp}\left(E_{\circ}^{\mathrm{H}_{2} / \perp}\right)$ is the total energy of the relaxed system including both the screw dislocation and $\mathrm{H}\left(\mathrm{H}_{2}\right), E_{\circ}^{\perp}$ is the total energy of the screw dislocation alone in the same computational system, and $E_{\mathrm{H}}$ is the reference energy for a single $\mathrm{H}$ atom. The latter is calculated with

$$
E_{\mathrm{H}}=\frac{1}{2}\left[E_{\circ}^{\mathrm{H}_{2}}-E_{\circ}\right]
$$

$E_{\circ}^{\mathrm{H}_{2}}$ being the total energy for $\mathrm{H}_{2}$ located in a $\mathrm{T}$ site, in the center of a bulk-like system, and $E_{\circ}$ the total energy of the same system but without hydrogen. Note that 
Table 2. Formation energies in eV for several stable $\mathrm{H}$ and $2 \mathrm{H}$ configurations in a screw dislocation in silicon, described in figures 2 and 3, calculated with the equations 1 and 2. They are computed relatively to an isolated screw dislocation and to $\mathrm{H}_{2}$ in a $\mathrm{T}$ site in bulk silicon. The configuration marked by a star corresponds to an undissociated $\mathrm{H}_{2}$ molecule.

\begin{tabular}{lllll}
\hline & $(\mathrm{a})$ & $(\mathrm{b})$ & $(\mathrm{c})$ & $(\mathrm{d})$ \\
\hline $\mathrm{H}$ in $\perp$ & -0.21 & -0.01 & 0.04 & 0.19 \\
\hline $2 \mathrm{H}$ in $\perp$ & -1.80 & -1.32 & 0.26 & $0.37^{*}$ \\
\hline
\end{tabular}

all these total energies have been obtained for similar computational systems, allowing for an efficient cancellation of surface contributions due to fixed boundary calculations.

The figure 2 shows the most stable configurations for a single $\mathrm{H}$ atom within the screw dislocation core. The corresponding formation energies are reported in the table 2 . Note that zero-point energy contributions have not been determined, since they have been shown to be very close for several H-Si configurations [11]. The lowest energy configuration is obtained when $\mathrm{H}$ is located at location $\mathrm{C}$ and is centered on a long bond (figure 2a). The distance between the two closest $\mathrm{Si}$ atoms increased from $2.54 \AA$ to $3.46 \AA$. Also, the $\mathrm{H}$ atom is not located exactly between two $\mathrm{Si}$ atoms, but is slightly displaced towards the center of the dislocation. This structure is similar to a $\mathrm{BC}$ configuration in bulk silicon, but because of the initially larger bond length, the accommodation of the hydrogen atom is much easier than in the bulk, lowering the total energy by $1.28 \mathrm{eV}$. This energy gain is very close to the value of $1.07 \mathrm{eV}$ computed by Scarle and Ewels for $\mathrm{H}$ in a $90^{\circ}$ partial dislocation [39]. $\mathrm{H}$ is therefore strongly bound to the dislocation core. Alternatively, one may consider that the presence of an hydrogen atom helps to relieve the large stress into the dislocation core. The formation energy is negative (one $\mathrm{H}$ atom forming $\mathrm{H}_{2}$ in bulk $\mathrm{T}$ site being the reference), indicating that atomic hydrogen in the dislocation core is energetically more favourable than molecular $\mathrm{H}_{2}$ in the bulk. This suggests that a dislocation could act as a dissociation center for the $\mathrm{H}_{2}$ molecule in a similar way as vacancies and interstitials [16].

The second best configuration (table 2) is obtained for an hydrogen atom located at $\mathrm{B}$, between two $\mathrm{Si}-\mathrm{Si}$ bonds (figure $2 \mathrm{~b}$ ). The hydrogen atom is asymmetrically positioned relatively to the closest $\mathrm{Si}$ atoms, with $\mathrm{Si}-\mathrm{H}$ distances of $1.65 \AA$ and $1.92 \AA$. The neighbour Si-Si bonds are enlarged to lengths of 2.50-2.53 $\AA$, with an increased tilting angle $\left(25^{\circ}-28^{\circ}\right)$, relatively to a screw dislocation core without hydrogen. The $\mathrm{H}$ atom does not remain exactly at the $\mathrm{B}$ location, but is sligthly displaced away from the dislocation center. This configuration and the previous one (figure 2a-b) correspond to an hydrogen atom located on the hexagon centered on the screw dislocation (figure 1). All attemps to obtain $\mathrm{H}$ inside the hexagon were unsuccessful, the system relaxing into one of the previously mentioned configurations. Finally, we found other possible configurations, higher in energy, for which the $\mathrm{H}$ atom is not anymore in the vicinity of the dislocation core (figure $2 \mathrm{c}-\mathrm{d}$ ). It is interesting to note that in that case the presence 


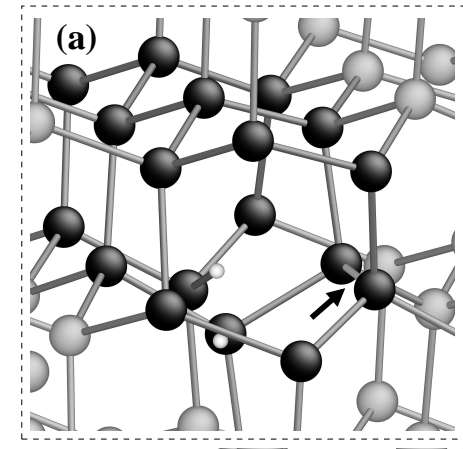

(c)

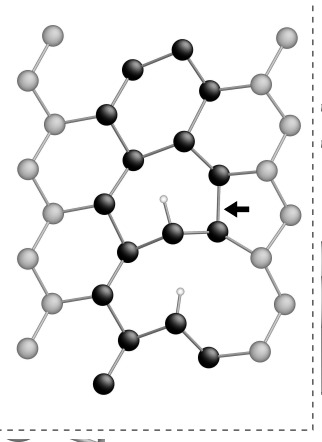

(b)

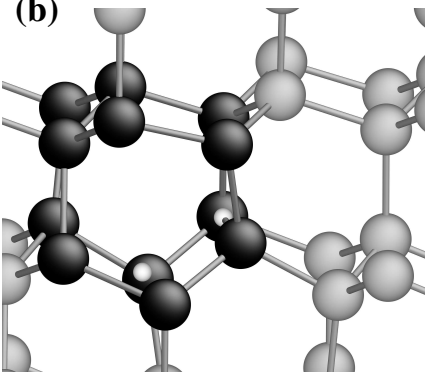

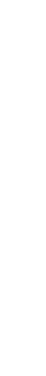

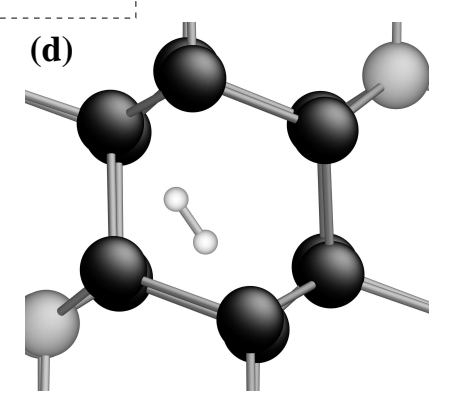

Figure 3. (a-d) Four possible stable configurations for two $\mathrm{H}$ atoms located in a screw dislocation core. The dislocation core is marked by black atoms. In the case of the most stable configuration $(\mathrm{a})$, the $([\overline{1} 1 \overline{1}])$ projection is also shown for clarity. The arrows point to a bond formed in the dislocation core after $\mathrm{H}$ insertion (see text for explanation).

of a single $\mathrm{H}$ is able to induce sizeable modifications of the core structure.

We have also studied the stability of molecular hydrogen in the screw dislocation core, starting from many possible initial $\mathrm{H}_{2}$ positions and orientations. We found that the $\mathrm{H}_{2}$ molecule is stable almost at the dislocation center, in a location seemingly equivalent to the $\mathrm{T}$ site in the cubic diamond structure (figure $3 \mathrm{~d}$ ). The $\mathrm{H}_{2}$ molecule length, $0.84 \AA$, is slightly larger than in the bulk $(0.80 \AA)$, and the orientation is close to [100]. However, the energy per $\mathrm{H}$ is $0.37 \mathrm{eV}$ higher compared to $\mathrm{H}_{2}$ in bulk, suggesting that the dihydrogen molecule is not attracted to the dislocation. For some initial configurations, the spontaneous dissociation of $\mathrm{H}_{2}$ into two single $\mathrm{H}$ atoms has been obtained. The final relaxed configuration is shown in the figure $3 \mathrm{~b}$, and corresponds to a large decrease energy of $1.32 \mathrm{eV}$ per $\mathrm{H}$ atom, relatively to $\mathrm{H}_{2}$ in the bulk. Like the configuration depicted in the figure $2 \mathrm{a}$, the structure is characterized by the breaking of one long bond at the $\mathrm{C}$ position. The two resulting dangling bonds are passivated by the hydrogen atoms, with $\mathrm{Si}-\mathrm{H}$ bond lengths of $1.53 \AA$. Within this geometry, the $\mathrm{H}$ atoms are expected to be non-interacting, the separation distance being $2.05 \AA$. This result confirms that the dissociation of the $\mathrm{H}_{2}$ molecule is favoured in the dislocation core.

Playing with the initial positions for $\mathrm{H}$ atoms, we have also tried to find other configurations for two distant $\mathrm{H}$ atoms with lower formation energy. Our best candidate is represented in the figure $3 \mathrm{a}$, and corresponds to a large energy decrease of $1.80 \mathrm{eV}$ 


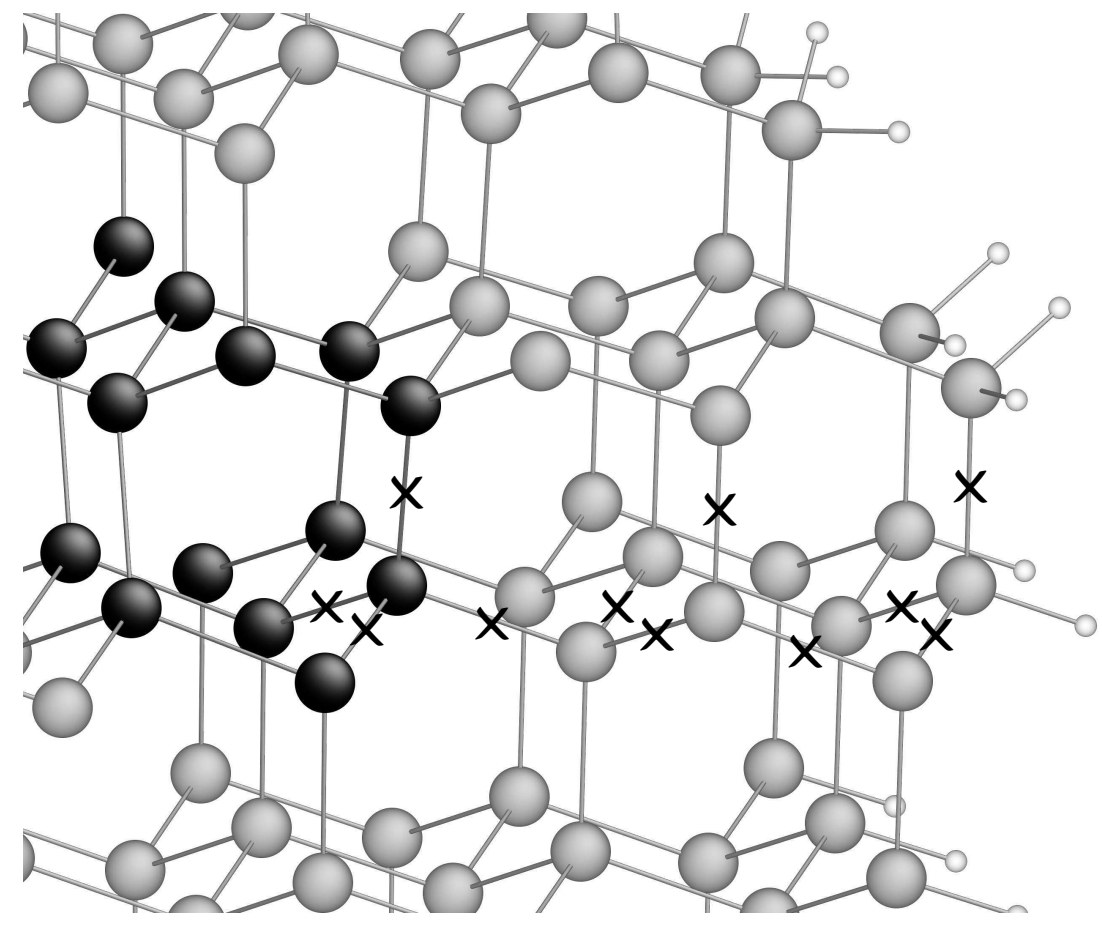

Figure 4. Different BC positions used for the determination of the interaction between $\mathrm{H}$ and the screw dislocation. Same color convention as in previous figures.

compared to one $\mathrm{H}_{2}$ molecule in a bulk $\mathrm{T}$ site (table 2). Compared to the previous structure, the hydrogen atoms are now bound to successive silicon atoms along [101]. We can not assert that this configuration is the absolute lowest energy one for two $\mathrm{H}$ in the dislocation core. It is also interesting to notice that a $\mathrm{Si}-\mathrm{Si}$ bond is created along [101] in the vicinity of the hydrogen atoms (marked by an arrow in the figure 3a). Because such a dimer is the base structural unit for the reconstructed screw dislocation core in the glide set [26], this result would hint that the insertion of hydrogen in the dislocation core could make easier the dislocation core reconstruction.

Our calculations for $\mathrm{H}$ and $\mathrm{H}_{2}$ clearly indicate that hydrogen segregation is favoured in the dislocation core. However, this effect is only obtained in the central hexagon of the core. Hence, if one $\mathrm{H}$ atom is located on the hexagon, while another is located in a nearby BC site (figure 3c), the energy is higher than for $\mathrm{H}_{2}$ in the bulk (table 2). Due to the small number of possible sites for $\mathrm{H}$ in the hexagon, the concentration of hydrogen in the dislocation core is therefore likely to be limited. However, we did not push further our investigations since a complete determination of all possible $\mathrm{H}$ configurations in the core, for an increasing $\mathrm{H}$ concentration, is out of the scope of this study.

\section{Interaction between screw dislocation and hydrogen}

We now focus on the behaviour of hydrogen in the elastic region, surrounding the dislocation core. An impurity in a material, like hydrogen in silicon in our case, is 
a source of stress. Therefore, it is expected to interact with the long-range elastic stress field associated with a dislocation. This is a classical textbook problem, and it has been shown that if the stress generated by the impurity is isotropic, and the crystal is elastically isotropic too, there is only an interaction with the hydrostatic component of the dislocation stress. Since a screw dislocation has no hydrostatic component, there should be no interaction with an isotropic source of stress in an isotropic crystal. This is no longer true when the crystal is elastically anisotropic, or when the stress field associated with the impurity is not isotropic $[38,40]$. The interaction is inversely proportional to the separation between the dislocation and the impurity [38]. Higher order interaction contributions at short dislocation-impurity separations, are also predicted in specific cases [38, 40].

For determining the interaction energy between $\mathrm{H}$ or $\mathrm{H}_{2}$, and the screw dislocation, we have computed the following quantities

$$
\begin{aligned}
& E_{I}^{\mathrm{H} / \perp}(d)=\left[E_{\circ}^{\mathrm{H} / \perp}(d)-E_{\circ}^{\perp}\right]-\left[E_{\circ}^{\mathrm{H}}(d)-E_{\circ}\right], \\
& E_{I}^{\mathrm{H}_{2} / \perp}(d)=\left[E_{\circ}^{\mathrm{H}_{2} / \perp}(d)-E_{\circ}^{\perp}\right]-\left[E_{\circ}^{\mathrm{H}_{2}}(d)-E_{\circ}\right],
\end{aligned}
$$

which look similar to equations 1 and 2, the only difference concerning the reference energy. For instance, $E_{\mathrm{H}}$ is now replaced by $\left[E_{\circ}^{\mathrm{H}}(d)-E_{\circ}\right]$ in the equation $4 . E_{\circ}^{\mathrm{H}}(d)$ is the total energy of the same cluster-like system as for $E_{\circ}^{\mathrm{H} / \perp}(d)$, with $\mathrm{H}$ located in a BC site characterized by the same separation $d$ relative to the cluster center, but without screw dislocation. This procedure allows us to define an effective interaction energy, vanishing for $d$ going to infinity, and also to cancel out spurious surface effects due to the finite size of our cluster system. The figure 4 shows most of the BC sites that have been considered in our investigations. In the case of $\mathrm{H}_{2}$, we employed the same method, except that the reference states were now the $\mathrm{T}$ sites.

The figure 5 represents the interaction energy variations as a function of $d$ for both $\mathrm{H}$ and $\mathrm{H}_{2}$. In the case of $\mathrm{H}$, it is found that $E_{I}^{\mathrm{H} / \perp}(d)$ is always negative, in agreement with an attractive interaction with the screw dislocation, and stronger for small separations. The interaction energy variation is best fitted with a simple inverse law $\alpha / d$, with $\alpha=-2.2 \mathrm{eV} \AA$, in accordance with an overall first-order interaction between the dislocation and the impurity. According to theory, such a behaviour could be observed only for an anisotropic source of stress. A configuration with $\mathrm{H}$ in the $\mathrm{BC}$ site is characterized by large displacements of the two Si atoms on both sides of $\mathrm{H}$ along the Si-H-Si axis exclusively, thus generating an anisotropic stress field. Comparing our data with the inverse law, we found a maximum dispersion of $0.3 \mathrm{eV}$. These small variations could be explained by the anisotropic character of silicon.

Surprinsingly, in the case of $\mathrm{H}_{2}$, no overall variation of the interaction energy was obtained (figure 5). The computed values are either close to zero or slightly positive, with an amplitude in the same range as the dispersion observed for $\mathrm{H}$. A possible explanation comes from the lattice distortions due to the insertion of an $\mathrm{H}_{2}$ molecule in the $\mathrm{T}$ site, which are rather small and generates a quasi isotropic stress field around the molecule. 


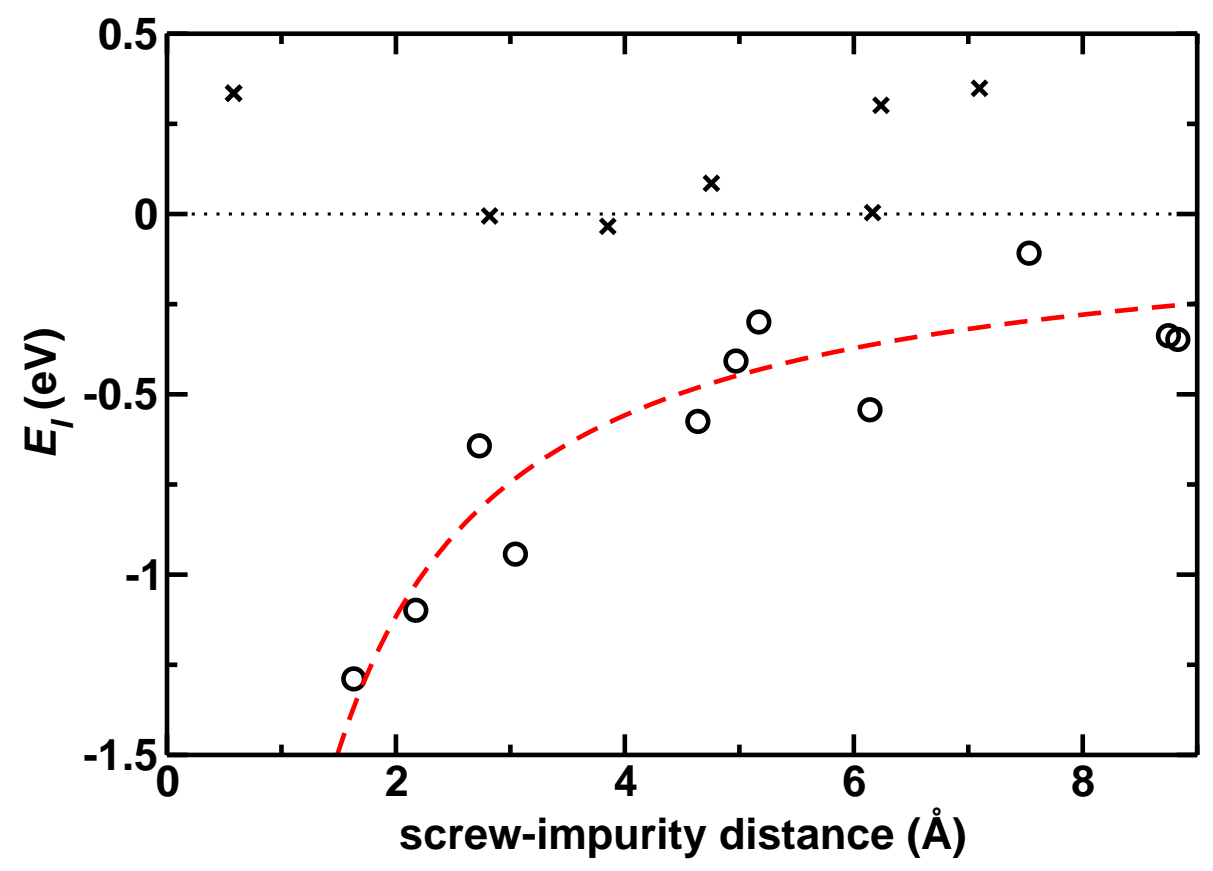

Figure 5. Interaction energies between $\mathrm{H}$ (circles) or $\mathrm{H}_{2}$ (crosses) and a screw dislocation in silicon, as a function of the separating distance. The dashed line corresponds to a simple inverse fit of the data for $\mathrm{H}$.

Consequently, there is no sizeable interaction between the screw dislocation and $\mathrm{H}_{2}$, in agreement with theory.

\section{Discussion}

To summarize, our first-principles calculations showed that atomic hydrogen is deeply attracted by a screw dislocation in silicon. The lowest energy configuration is obtained for $\mathrm{H}$ located in a $\mathrm{BC}$ site, between silicon atoms belonging to the dislocation core. Therefore, since atomic hydrogen is a very fast diffuser, it is expected to segregate in the dislocation core if available in large amount. Conversely, molecular hydrogen is not attracted by the dislocation, but our results indicated that if a $\mathrm{H}_{2}$ molecule diffuses in the dislocation core, it could spontaneously dissociate, freeing an excess energy of at most $1.8 \mathrm{eV}$. The computation of the interaction energy between atomic hydrogen and the screw dislocation revealed that it is inversely proportional to the impurity-dislocation separation. This is in agreement with classical theory if one takes into account that hydrogen in BC site generates an anisotropic stress field. In the case of molecular hydrogen, there is no noticeable interaction, which can be explained by the small and isotropic lattice distortion associated with $\mathrm{H}_{2}$ in a $\mathrm{T}$ site.

In this work, the mobility of $\mathrm{H}$ along the dislocation line has not been investigated. In bulk silicon, earlier studies have shown that the barrier for diffusion is small $[1,5$, $6,7,8,9,10]$. It is difficult to predict whether the mobility in the dislocation core will 
be enhanced or reduced. On the one hand, the stability of $\mathrm{H}$ is greatly improved in the core, thus suggesting that migration barrier could be larger. On the other hand, the lattice distortions in the dislocation core could make the diffusion easier, by lowering the saddle states, or opening new migration paths with low energy. This point requires further investigations to be clarified.

An interesting question concerns the transferability of our results in the case of other dislocation cores in silicon, such as $30^{\circ}$ and $90^{\circ}$ partial or $60^{\circ}$ perfect dislocations. To consider all possible core structures is a formidable task, and it is tempting to try to extract transferable information from the single case examined here. First, we note that all dislocations in silicon are characterized by the stretching and bending of bonds in the core. Second, our calculations showed that moderate distortions of silicon bonds, such as those present in the screw dislocation core are enough to largely improve the stability of $\mathrm{H}$. In fact, the best configuration for $\mathrm{H}$ in the dislocation core corresponds to a formation energy $1.28 \mathrm{eV}$ lower than $\mathrm{H}$ in bulk $\mathrm{BC}$ site. In addition, the presence of an initial hydrogen in the core seems to further reduce the formation energy. Therefore, even if the core does not include dangling bonds, it is likely that the stability of $\mathrm{H}$ is largely improved by a dislocation in silicon in any cases. Nevertheless, it is difficult to assert whether the predicted "catalytic" effect leading to the dissociation of $\mathrm{H}_{2}$ in the screw dislocation could be active for other dislocation cores. Another possible effect of the presence of hydrogen is an improvement of the stability of the dislocation core in specific cases [41].

Finally, the influence of hydrogen on the mobility of the screw dislocation remains to be examined. Such a study is difficult to make, since one has to determine energies associated with formation and migration of kinks in presence of hydrogen. Parts of the answer have been given by Ewels and co-workers [24] in the case of the $90^{\circ}$ partial dislocation. Regarding the screw dislocation, it has been shown that kinks are characterized by dangling bonds and are highly mobile [42]. While it is difficult to predict how hydrogen will modify the kink formation, one might presume that hydrogen could passivate the dangling bonds, thus shutting off the fast kink migration. Additional investigations are necessary for confirmation and better understanding.

\section{Acknowledgments}

This work was supported by the SIMDIM project under contract $\mathrm{N}^{\circ}$ ANR-06-BLAN250 . 


\section{References}

[1] M. Stavola. Hydrogen diffusion and solubility in c-Si, chapter 9.8, page 511. Number 20 in EMIS Datareviews. INSPEC, London, 1999.

[2] B. Terreault. Hydrogen blistering of silicon: Progress in fundamental understanding. Phys. Status Solidi A, 204:2129, 2007.

[3] S. Reboh, M. F. Beaufort, J.-F. Barbot, J. Grilhé, and P. F. P. Fichtner. Orientation of h platelets under local stress in si. Appl. Phys. Lett., 93:022106, 2008.

[4] G. F. Cerofolini, F. Corni, S. Frabboni, C. Nobili, G. Ottaviani, and R. Tonini. Hydrogen and helium bubbles in silicon. Mater. Sci. Eng. Reports, 27:1, 2000.

[5] A. Van Wieringen and N. Warmoltz. Physica, 22:849, 1956.

[6] P. Deák, L. C. Snyder, and J. W. Corbett. State and motion of hydrogen in crystalline silicon. Phys. Rev. B, 37(12):6887, 1988.

[7] F. Buda, G. L. Chiarotti, R. Car, and M. Parrinello. Proton diffusion in crystalline silicon. Phys. Rev. Lett., 63(3):294, 1989.

[8] K. J. Chang and D. J. Chadi. Hydrogen bonding and diffusion in crystalline silicon. Phys. Rev. $B, 40(17): 11644,1989$.

[9] C. G. van de Walle, P. J. H. Denteneer, Y. Bar-Yam, and S. T. Pantelides. Theory of hydrogen diffusion and reactions in crystalline silicon. Phys. Rev. B, 39(15):10791, 1989.

[10] P. E. Blöchl, C. G. van de Walle, and S. T. Pantelides. First-principles calculations of diffusion coefficients: hydrogen in silicon. Phys. Rev. Lett., 64(12):1401, 1990.

[11] C. G. van de Walle. Energies of various configurations of hydrogen in silicon. Phys. Rev. B, 49(7):4579, 1994.

[12] A. J. Morris, C. J. Pickard, and R. J. Needs. Hydrogen/silicon complexes in silicon from computational searches. Phys. Rev. B, 78:184102, 2008.

[13] S. K. Estreicher, M. A. Roberson, and Dj. M. Maric. Hydrogen and hydrogen dimers in c-c, si, ge, and $\alpha$-sn. Phys. Rev. B, 50(23):17018, 1994.

[14] F. A. Reboredo, M. Ferconi, and S. T. Pantelides. Theory of the nucleation, growth, and structure of hydrogen-induced extedend defects in silicon. Phys. Rev. Lett., 82(24):4870, 1999.

[15] M. Stavola. Hydrogen-containing point defects in c-Si, chapter 9.9, page 522. Number 20 in EMIS Datareviews. INSPEC, London, 1999.

[16] S. K. Estreicher, J. L. Hastings, and P. A. Fedders. Defect-induced dissociation of $\mathrm{h}_{2}$ in silicon. Phys. Rev. B, 57(20):R12663, 1998.

[17] A. Antonelli, J.F.Justo, and A. Fazzio. Arsenic segregation, pairing and mobility on the cores of partial dislocations in silicon. J. Phys.: Condens. Matter, 14:12761, 2002.

[18] A. Antonelli, J. F. Justo, and A. Fazzio. Interaction of as impurities with $30^{\circ}$ partial dislocations in si: An ab initio investigation. J. Appl. Phys., 91(9):5892, 2002.

[19] I. Yonenaga. Dislocation-impurity interaction in si. Mater. Sci. Eng. B, 124-125:293, 2005.

[20] F. Bernardini and L. Colombo. Interaction of doping impurities with the $30^{\circ}$ partial dislocation in sic: An ab initio investigation. Phys. Rev. B, 72:085215, 2005.

[21] C. Li, Q. Meng, K. Zhong, and C. Wang. Computer simulation of the $60^{\circ}$ dislocation interaction with vacancy cluster in silicon. Phys. Rev. B, 77:045211, 2008.

[22] C. Li and Q. Meng. Computer simulation of the vacancy defects interaction with shuffle dislocation in silicon. Superlatt. Microstruc., 45:1, 2009.

[23] Y. Yamashita, F. Jyobe, Y. Kamiura, and K. Maeda. Hydrogen enhanced dislocation glides in silicon. Phys. Stat. Sol. (a), 171:27, 1999.

[24] C. P. Ewels, S. Leoni, M. I. Heggie, P. Jemmer, E. Hernndez, R. Jones, and P. R. Briddon. Hydrogen interaction with dislocations in si. Phys. Rev. Lett., 84(4):690, 2000.

[25] M. I. Heggie, S. Jenkins, C. P. Ewels, P. J̈emmer, R. Jones, and P. R. Briddon. Theory of dislocations in diamond and silicon and their interaction with hydrogen. J. Phys.: Condens. Matter, 12:10263, 2000. 
[26] C.-Z. Wang, J. Li, K.-M. Ho, and S. Yip. Undissociated screw dislocation in si: Glide or shuffle set? Appl. Phys. Lett., 89:051910, 2006.

[27] L. Pizzagalli, J. L. Demenet, and J. Rabier. Theoretical study of pressure effect on the dislocation core properties in semiconductors. Phys. Rev. B, 79:045203, 2009.

[28] L. Pizzagalli, P. Beauchamp, and J. Rabier. Undissociated screw dislocations in silicon: Calculations of core structure and energy. Philos. Mag. A, 83:1191, 2003.

[29] W. Cai, V.V. Bulatov, J. Chang, J. Li, and S. Yip. Dislocation core effects on mobility. In F.R.N. Nabarro and J.P. Hirth, editors, Dislocations in Solids, volume 12, chapter 64, page 1. North Holland, 2004.

[30] J. Rabier, P. Cordier, J. L. Demenet, and H. Garem. Plastic deformation of si at low temperature under high confining pressure. Mater. Sci. Eng. A, 309-310:74, 2001.

[31] J. Rabier. High-stress plasticity and the core structures of dislocations in silicon. Phys. Stat. Sol. (a), 204(7):2248, 2007.

[32] J. Rabier, L. Pizzagalli, and J.-L. Demenet. Dislocations in silicon at high stress. In L. Kubin and J. P. Hirth, editors, Dislocation in solids, volume 16, chapter 93, page 47. Elsevier, 2010.

[33] D. Sánchez-Portal, P. Ordejón, E. Artacho, and J.M. Soler. Density-functional method for very large systems with lcao basis sets. Int. J. Quantum Chem., 65:453, 1997.

[34] E. Artacho, D. Sánchez-Portal, P. Ordejón, García A., and J. M. Soler. Linear-scaling ab-initio calculations for large and complex systems. Phys. Status Solidi b, 215:809-817, 1999.

[35] J. M. Soler, E. Artacho, J. D. Gale, A. García, J. Junquera, P. Ordejón, and D. Sánchez-Portal. The siesta method for $a b$ initio order $-n$ materials simulation. J. Phys.: Condens. Matter, 14:2745, 2002.

[36] J. P. Perdew, K. Burke, and M. Ernzerhof. Generalized gradient approximation made simple. Phys. Rev. Lett., 77(18):3865, 1996.

[37] Y. Okada. Diamond cubic Si: structure, lattice parameter and density, chapter 3.1, page 91. Number 20 in EMIS Datareviews. INSPEC, London, 1999.

[38] J. P. Hirth and J. Lothe. Theory of dislocations. Wiley, New York, 1982.

[39] S. Scarle and C.P. Ewels. Kinetic monte carlo and density functional study of hydrogen enhanced dislocation glide in silicon. Eur. Phys. J. B, 51:195, 2006.

[40] F. R. N. Nabarro. Theory of crystal dislocations. Dover, New York, 1987.

[41] L. Pizzagalli, J. Godet, and S. Brochard. Glilssile dislocations with transient cores in silicon. Phys. Rev. Lett., 103:065505, 2009.

[42] L. Pizzagalli, A. Pedersen, A. Arnaldsson, H. Jónsson, and P. Beauchamp. Theoretical study of kinks on screw dislocation in silicon. Phys. Rev. B, 77:064106, 2008. 